\title{
Kernos
}

Revue internationale et pluridisciplinaire de religion grecque antique

12| 1999

Varia

\section{HINZ, Der Kult von Demeter und Kore auf Sizilien und in der Magna Graecia}

\section{Véronique Suys}

\section{OpenEdition \\ Journals}

Édition électronique

URL : http://journals.openedition.org/kernos/734

DOI : $10.4000 /$ kernos.734

ISSN : 2034-7871

\section{Éditeur}

Centre international d'étude de la religion grecque antique

\section{Édition imprimée}

Date de publication : 1 janvier 1999

Pagination : $308-310$

ISSN : 0776-3824

\section{Référence électronique}

Véronique Suys, « V. HINz, Der Kult von Demeter und Kore auf Sizilien und in der Magna Graecia », Kernos [En ligne], 12 | 1999, mis en ligne le 13 avril 2011, consulté le 11 mars 2021. URL : http:// journals.openedition.org/kernos/734 ; DOI : https://doi.org/10.4000/kernos.734 
Valentina Hinz, Der Kult von Demeter und Kore auf Sizilien und in der Magna Graecia, Wiesbaden, Ludwig Reichert Verlag, 1998. 1 vol. $22 \times 29 \mathrm{~cm}$, 262 p., 69 fig. (Deutsches Archäologisches Institut Rom. Palilia, 4). ISBN : 389500-052-3.

Cet ouvrage est le fruit d'une thèse de doctorat soutenue au début de l'année 1995. Malgré l'ampleur des témoignages archéologiques relatifs au culte de Déméter et de Koré en Grande Grèce et surtout en Sicile, force est de reconnaître que jusqu'à présent, ils n'ont pas reçu l'attention qu'ils méritent. En effet, constate l'A., les études consacrées aux deux déesses dans ces régions se fondent presque exclusivement sur les textes, n'utilisant les données matérielles qu'à titre illustratif. Pour combler cette lacune, V. Hinz se propose d'étudier systématiquement les différents sanctuaires connus des deux déesses, insistant sur la nécessité de partir d'une approche locale, qui permette de dégager les caractéristiques propres à chaque lieu de culte, tout en le considérant dans ses rapports avec son environnement. Fondée essentiellement sur les données archéologiques, l'enquête prendra cependant en compte les autres types de source. Une synthèse finale visera à définir le caractère du culte des deux déesses en Sicile et en Italie du Sud, à en retracer l'expansion et l'évolution, et, finalement, à en déterminer les spécificités par rapport à leurs cultes dans le reste du monde grec.

L'étude proprement dite des sanctuaires est précédée de deux chapitres abordant des questions plus générales. Dans le premier, qui porte sur les sources écrites, l'A. examine d'abord les différentes thèses défendues par la recherche moderne pour expliquer le lien tout particulier qui, aux yeux des anciens, unissait les deux déesses à la Sicile. Des trois explications proposées, à savoir l'assimilation avec une déesse précoloniale de nature analogue, l'utilisation du culte à des fins politiques par les tyrans, la fertilité particulière de la Sicile, V. Hinz retient la dernière, après avoir écarté les deux autres sur la base d'arguments qui semblent fondés. Le point suivant concerne les circonstances dans lesquelles fut élaborée, à partir des différentes traditions locales, la version sicilienne du rapt de Koré. Enfin, l'A. passe en revue les fêtes et les épiclèses mentionnées par les textes. Celles-ci révèlent un culte essentiellement axé sur la fertilité, tandis qu'à peu près aucune allusion n'est faite à des mystères. L'enquête archéologique viendra corroborer ces indications. Le second chapitre, plus étendu, est une présentation des témoignages archéologiques. Après avoir évoqué les problèmes liés à l'identification d'un sanctuaire et défini les principes méthodologiques à respecter dans ce domaine, l'A. examine successivement les terres cuites votives, la céramique et les autres types d'objets, la situation topographique des sanctuaires, le témenos et son architecture, le rite et la pratique sacrificielle. Les terres cuites, dont les types les plus caractéristiques sont les statuettes au porcelet et les bustes, font l'objet de l'exposé le plus développé. L'étude est menée avec clarté et rigueur, dans une perspective chronologique qui fait bien apparaître l'évolution des pratiques cultuelles.

On en arrive ainsi à l'examen des sites, qui constitue le corps de l'ouvrage. La première partie concerne la Sicile, la seconde l'Italie du sud. Chaque cité abordée fait l'objet d'une brève introduction historique et, quand il y a lieu, d'une présen tation succincte des témoignages textuels et numismatiques. Un plan situe l'en semble des sanctuaires de la polis, y compris ceux des autres divinités. Des plans, généralement très clairs, sont également fournis pour la plupart des lieux de culte étudiés. Plutôt que d'analyser séparément les différents éléments constitutifs du sanctuaire (objets votifs, structures, etc.), comme on le fait souvent, V. Hinz les étudie en parallèle à l'intérieur de chaque époque. Le grand avantage de cette 
démarche est de donner l'aperçu le plus complet possible d'un sanctuaire et de ses pratiques cultuelles au sein d'une époque déterminée. Les études portant sur les sanctuaires particulièrement importants se terminent par une mise en perspective qui replace le culte dans le contexte historique, social, religieux, etc., de la polis dont il dépend.

La conclusion présente une très bonne synthèse des résultats obtenus. Dans une première partie, la plus substantielle, l'A. brosse le tableau du culte et de son évolution. Elle distingue cinq périodes, allant de la deuxième moitié du vir ${ }^{\mathrm{e}} \mathrm{s}$, époque de la fondation des plus anciens sanctuaires connus des déesses, au $\operatorname{mi}^{\mathrm{e}} \mathrm{s}$. av. J.-C., après lequel le culte ne semble subsister que dans les sanctuaires monumentaux de Sicile, ce qui contraste avec l'image florissante qu'en donnent les sources littéraires. Les trois sections suivantes portent respectivement sur les participants au culte, l'expansion du culte chez les Grecs de l'ouest, les déesses chez les Carthaginois. Enfin, la dernière partie aborde la question de la spécificité du culte sicilien de Déméter et de Koré. Si, dans ses caractéristiques essentielles, il ne s'écarte guère des autres cultes démétriaques, il présente cependant, à partir de l'époque classique, certains aspects qui lui sont propres : une uniformisation marquée des figurines votives, l'aménagement de sanctuaires dans les quartiers d'habitation et dans des grottes, la tendance à la monumentalisation.

L'ouvrage contient un résumé en italien, ainsi que quatre index.

V. Hinz a réalisé un travail sérieux, qui apporte une contribution utile à notre connaissance du culte de Déméter et de Koré. L'A. exploite les témoignages archéologiques avec intelligence et prudence, en évitant le double écueil de la surinterprétation et, à l'inverse, de la simple énumération des données.

Certaines déceptions attendent toutefois le lecteur. D'abord au sujet de la présentation. Sur les plans des cités auraient dû figurer non seulement les sanctuaires, mais aussi les autres lieux importants. Les renvois bibliographiques manquent souvent de précision. Ainsi, l'A. relève que les ossements d'animaux mis au jour au sanctuaire de la Malophoros à Sélinonte appartiennent principalement à des caprins, fait important, notamment pour l'interprétation de l'épiclèse. Or, aucune note ne figurant à cet endroit, le lecteur qui souhaiterait approfondir la question se voit contraint de consulter un à un les rapports de fouille dont la liste est fournie au début de l'exposé. Il est regrettable aussi que les épiclèses divines ne soient pas reprises dans l'index, et qu'aucun texte ne soit cité. À côté de ces inconvénients liés à la présentation, l'ouvrage contient encore un certain nombre de lacunes et d'imprécisions, tandis que plusieurs points pouvaient être plus approfondis. Pour en revenir au sanctuaire de Déméter Malophoros, on apprend que les statuettes de l'époque classique, offrant un répertoire plus étendu que dans les autres sites étudiés, portent des attributs très variés, dont le porcelet n'est qu'un exemple. Or, l'A. ne dit pas en quoi consistent ces attributs, et d'autre part, rien ne permet au lecteur d'évaluer la proportion des figurines au porcelet par rapport aux autres, alors qu'il y avait moyen de citer des chiffres ( $c f$. p. ex. M. DewaIlly, Les statuettes aux parures du sanctuaire de la Malophoros à Sélinonte, Naples, 1992, p. 150-152). D'autre part, à propos des defixiones du même sanctuaire ( $\mathrm{v}^{\mathrm{e}} \mathrm{s}$. av. J.-C.), on lit qu'elle sont adressées aux divinités des Enfers (p. 150). Or, un seul de ces huit textes porte le nom d'une divinité, et il s'agit d'áyvì $\theta \in o ́ s$, ce qu'il fallait mentionner ( $c f$. L. Dubors, Inscriptions grecques dialectales de Sicile, Rome, 1989, n³3-40, p. 43-54). Par ailleurs, les defixiones ont été découvertes au nord du sanctuaire, au milieu d'un champ de stèles dédiées à Zeus Meilichios, en face du témenos de ce même dieu. Or, si l'on en croit M. Dewailly (op. cit., p. 36-37, avec d'autres références), les fouilles récentes ont permis d'établir que, contrairement à ce que l'on avait cru au début, le dit témenos ne faisait 
pas partie du sanctuaire de Déméter. La discussion vaut la peine d'être signalée car il n'est pas indifférent pour l'étude du culte de la déesse de savoir si oui ou non Zeus Meilichios était honoré dans son sanctuaire, et, de même, si le lieu d'enfouissement des plaques de défixions en faisait partie ou non. Or, non seulement V. Hinz ne fait pas la moindre allusion au problème, mais encore, sa position à ce sujet n'est pas claire. En effet, elle n'évoque Zeus Meilichios que dans la synthèse finale sur le sanctuaire de la Malophoros, et non au moment de sa description proprement dite. Mais en même temps, les defixiones sont présentées comme issues du complexe, et, de même, le plan peut laisser croire que le témenos de Zeus en fait partie. Un autre point concerne les pinakes du sanctuaire de Perséphone à Locres, ces fameuses plaques illustrées de scènes religieuses, qui constituent notre principale source d'informations sur le culte locrien de la déesse (à ce sujet, voir p. ex., Chr. Sounvinou-Inwood, Persephone and Apbrodite at Locri: A Model for Personality Definitions in Greek Religion, in JHS, 98 [1978], p. 101-121). Étant donné l'intérêt de ces documents et les problèmes d'interprétation qu'ils soulèvent, une étude à leur sujet avait toute sa place dans un ouvrage tel que celui-ci. Aussi le lecteur est-il très surpris de n'y voir consacrées que quelques lignes et de n'en trouver aucune illustration. Signalons enfin une question d'interprétation. L'A. considère que la standardisation des statuettes de terre cuite apparaissant au $\mathrm{v}^{e} s$. pourrait signifier que, tout comme les Thesmophories d'Athènes, les fêtes siciliennes étaient à cette époque réservées aux citoyennes (p. 227). Si le fait n'est pas impossible en lui-même, surtout dans les circonstances politiques et sociales du moment ( $c f$. p. 226-227), cependant, on ne comprend pas bien en quoi l'uniformisation des figurines votives le suggère.

Ces critiques ne doivent pas occulter les qualités de l'ouvrage, qui deviendra certainement une référence pour le culte de Déméter et de Koré.

Véronique Suys (Université Catholique de Louvain Facultés universitaires Saint-Louis)

Kentron. Revue du monde antique et de psycbologie bistorique, 14 (1998)= Actes des travaux du Groupe de Recherche "Mythe, bistoire et psychanalyse" sur Dionysos et l'énigme des Bacchantes (1995-1998). ISSN : 07650590 .

Ainsi qu'en témoigne la bibliographie dressée par G. Karsai en fin d'ouvrage, la littérature scientifique suscitée par les Bacchantes d'Euripide est particulièrement abondante et n'a cessé de crôitre ces dernières années. Fruit des travaux du groupe de recherches Mytbe, Histoire et Psychanalyse, cette publication comptera dans l'histoire du dionysisme tant elle renouvelle, par l'originalité de ses perspectives pluridisciplinaires, l'étude du texte d'Euripide. Plusieurs contributions sont d'ailleurs consacrées au long parcours suivi par Les Bacchantes, depuis sa création, en 405 (Fr. Jouan), jusqu'à sa survivance au Moyen Âge dans le Christos Pascbôn (J.-M. Mathieu), et sa toute récente mise en scène par I. Bergman (P. Legangneux).

Fr. Jouan, dans Une introduction au mythe des Bacchantes, montre comment s'est constituée la tragédie d'Euripide, et quelles furent ses principales voies de transmission philologique. Dans une même optique, l'article de J. Bottéro souligne l'existence d'éléments similaires dans un mythe mésopotamien récemment découvert et traduit qui, sur le célèbre modèle de la Descente d'Ishtar aux Enfers, relate la catabase de Nin-gesh-zi-da, probablement un dieu du vin; J. Bottéro ouvre ainsi la voie à la prudente comparaison avec les mythes proche-orientaux. 